\title{
cubiertas colgantes de hormigón República Argentina
}

JORGE W. MAGALDI, Ingeniero Civil

\section{sinopsis}

Estas cubiertas colgantes han surgido como resultado de los innumerables estudios realizados para cubrir grandes espacios de form

La estructura portante de estas láminas se constituye con pórticos de hormigón armado, que apoyan sobré cimentacion material. Dichos porticos pueden ser prefabriproyecto que previamente se haya realizado para cada caso particular.

La estructura así construida se puede rematar mediante dos soluciones de láminas colgantes: Ambas combinan la otra, de hormigón armado. les complementarios, con los que presentan ventajas de estanquidad, minimo mantenimien to, facilidad para la colocación de aislamiento térmico y cielorraso, junto a la propiedad de me cubriendo grandes espacios y permitir un diseño arquitectónico de lineas muy avanzadas.
$886-31$

La función arquitectónica de cerrar un espacio se ha podido realizar en el transcurso de los tiempos utilizando las más diversas técnicas. Con la aparición del hierro como material constructivo aplicado a las modernas estructuras, el problema de cubrir el espacio y sustentar ese cerramiento se ha visto muy simplificado. Lo que antes estaba limitado por los materiales, imposibilitando alcanzar mayores luces, se supera ahora con las grandes posibilidades de las estructuras metálicas y con el empleo del hormigón armado.

Las láminas colgantes son el resultado del esfuerzo realizado para conseguir rematar una estructura de grandes luces con una cubierta idónea.

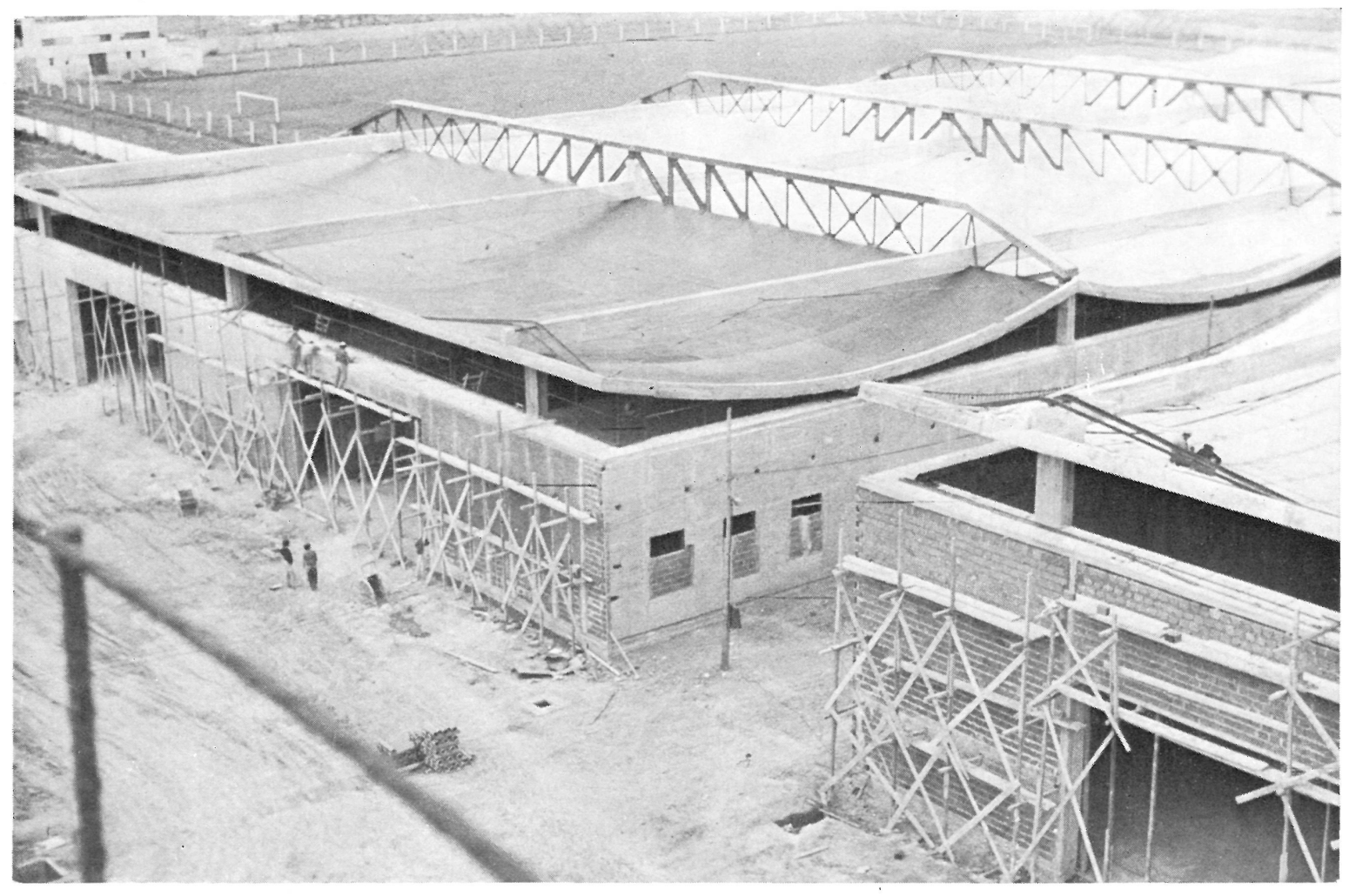

Láminas colgantes terminadas. 


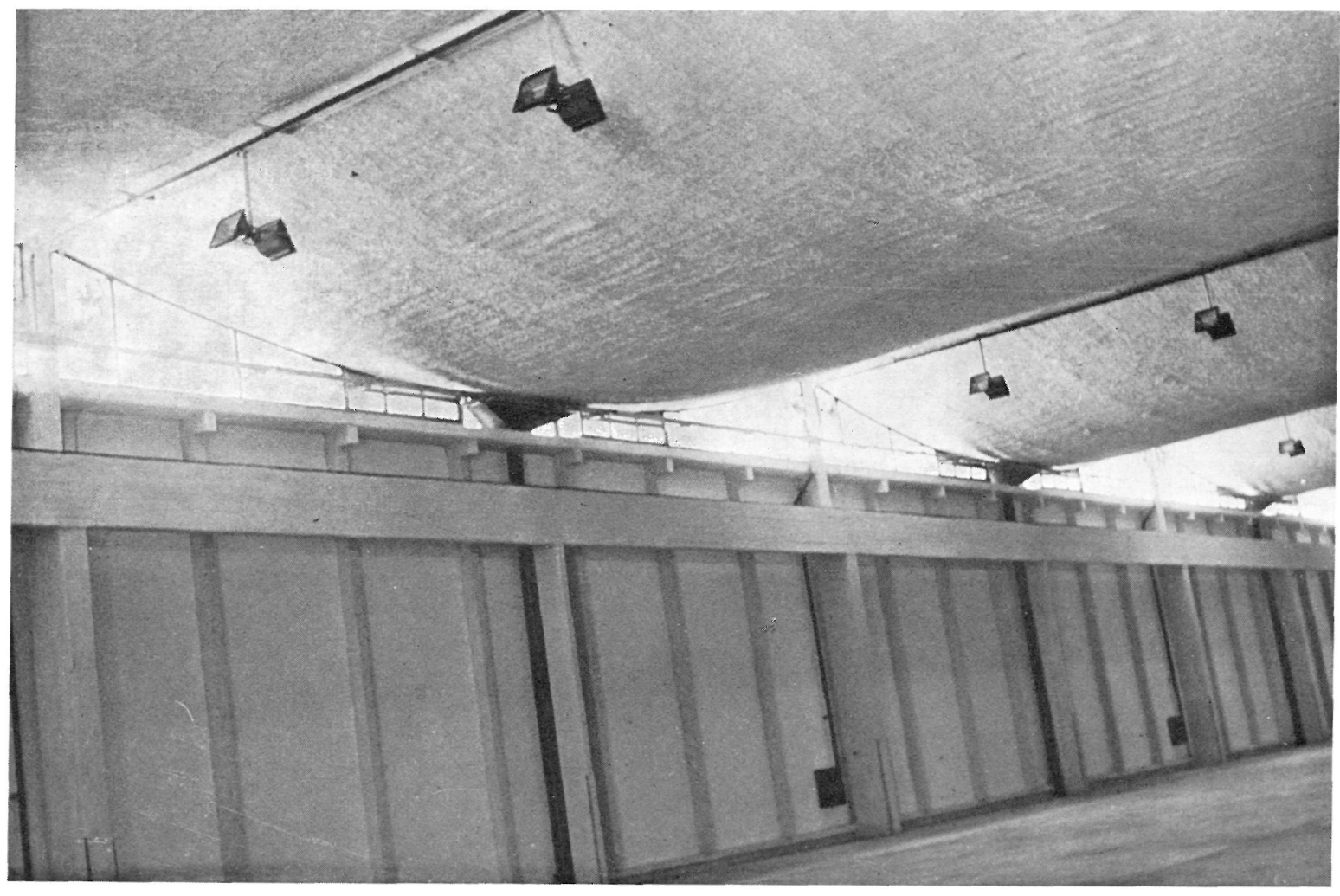

Vista interior.

La estructura portante de estas láminas se constituye con pórticos de hormigón armado, vibrado y de alta resistencia, sustentados sobre cimentaciones del mismo material. Los pórticos pueden ser prefabricados, dependiendo su diseño definitivo de las características de la planta a cubrir y de las demás condiciones funcionales de cada obra en particular. Estos pórticos se calculan para soportar, además de la carga de la cubierta, todos los empujes de viento sobre el edificio, las cargas de paredes perimetrales, los puentes-grúa que se pudieran instalar, etc. En definitiva, estos elementos constructivos, formados por sus bases, columnas, vigas inclinadas de borde y puntales, aseguran una total rigidez derivada del hormigón armado del que están construidos. También hay que añadir, como ventajas, su indestructibilidad y los casi nulos gastos de mantenimiento, por la gran limpieza de acabado del material, así como una alta seguridad ante los siniestros.

Estructura resistente soporte de la lámina.
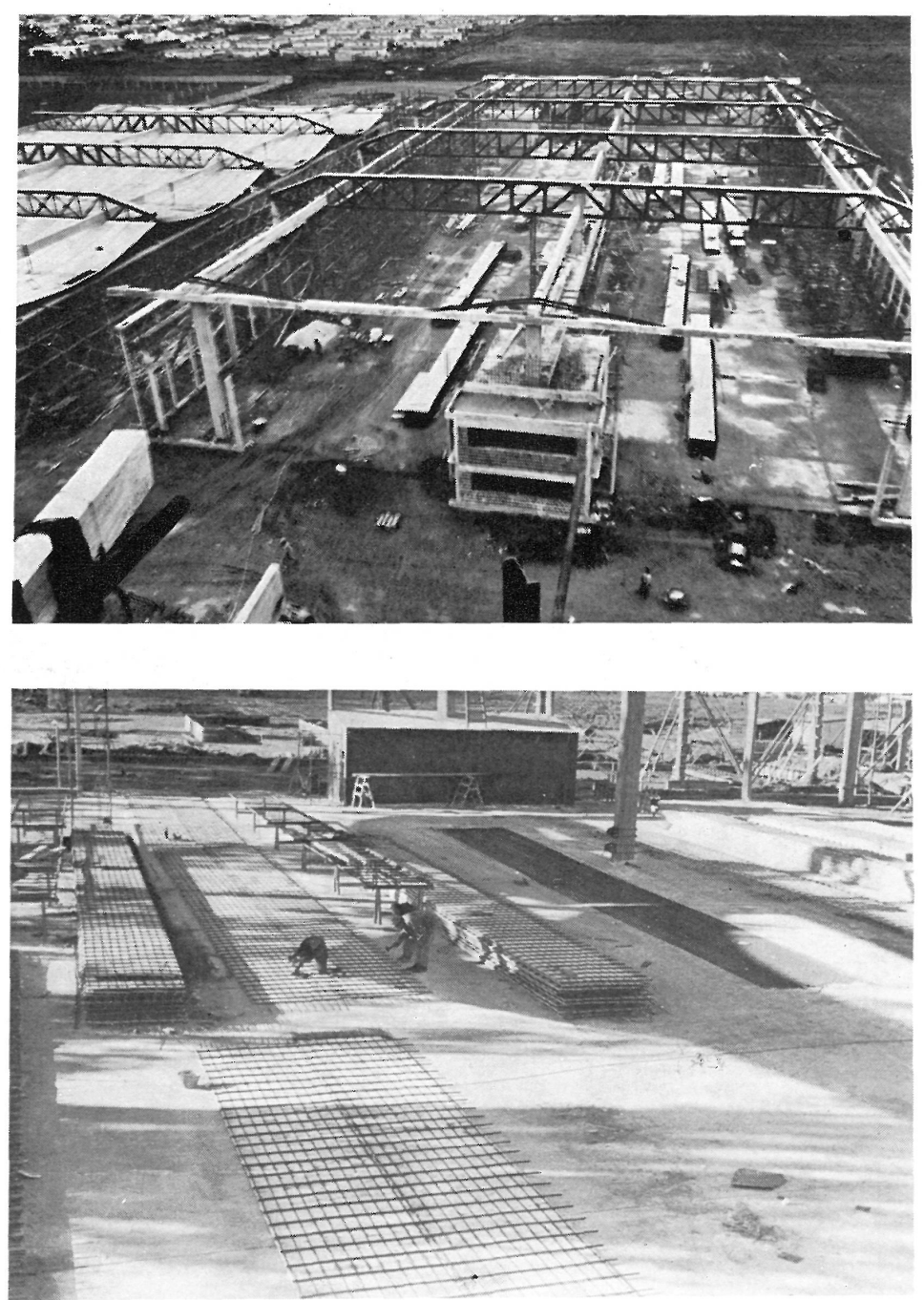


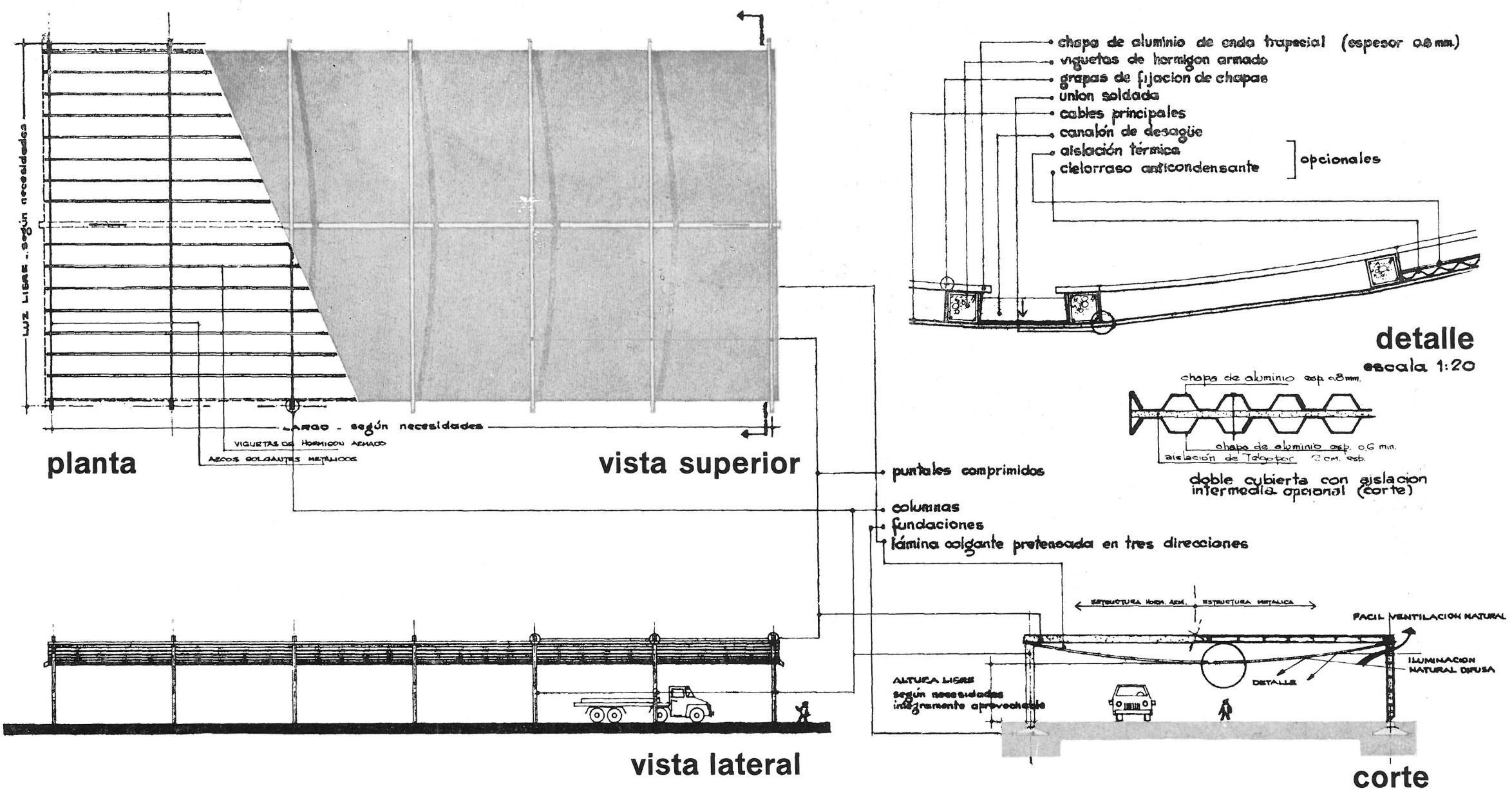

๓ 


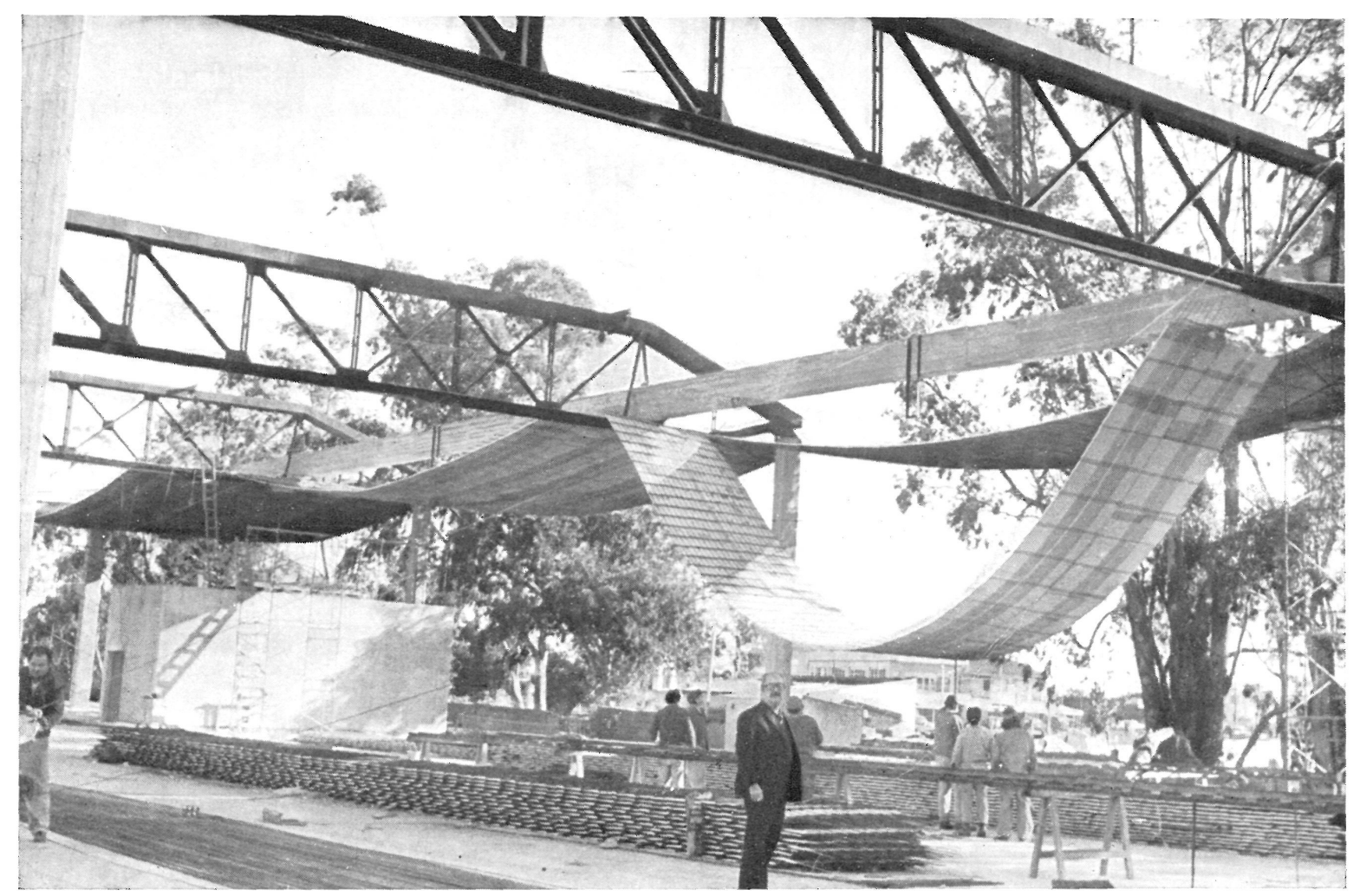

Montaje de láminas colgantes.

Sobre la estructura portante, así realizada, se puede construir la de cubierta, mediante dos soluciones de lámina colgante: una metálica y otra de hormigón armado.

En el primer caso, la estructura portante de la cubierta se forma por una serie de cables o hierros redondos totalmente tensados. Estos soportan el conjunto de viguetas de hormigón ar. mado, sobre las que van fijadas las chapas plegadas de aluminio, de $0,8 \mathrm{~mm}$ de espesor y largo adecuado para evitar totalmente las juntas transversales, que forman la cubierta propiamente dicha. El conjunto está pretensado hacia abajo y en el plano de la cubierta, asegurando así una total estabilidad ante el efecto del viento.

Los cables toman, de manera natural, la forma de catenarias suaves, con una flecha máxima de $1 / 20$ de la luz entre apoyos. Resulta así una cubierta casi plana, con su convexidad hacia abajo y con los bordes levantados, lo que favorece la ventilación e iluminación naturales.

El aspecto interno es sumamente claro y limpio. Los únicos elementos que necesitan conservación son los cables principales, colocados cada $2 \mathrm{~m}$ aproximadamente, y que, al estar separados $20 \mathrm{~cm}$ de las chapas plegadas de cubierta, quedan fácilmente accesibles. También se garantiza un perfecto desagüe pluvial por su forma y pendientes, ya que las chapas vierten el agua, por los canales que conforman su plegado, sobre un gran canalón central de zinc reforzado que la conduce libremente hacia los frentes.

Esta cubierta presenta características de estanquidad superiores a las de todas sus similares, poseyendo además unas buenas condiciones para reflejar los rayos solares, ventajas que la hacen mejorar en su habitabilidad. Estas características se completan con la facilidad y economía para instalar un buen aislamiento térmico y un cielorraso anticondensante. 

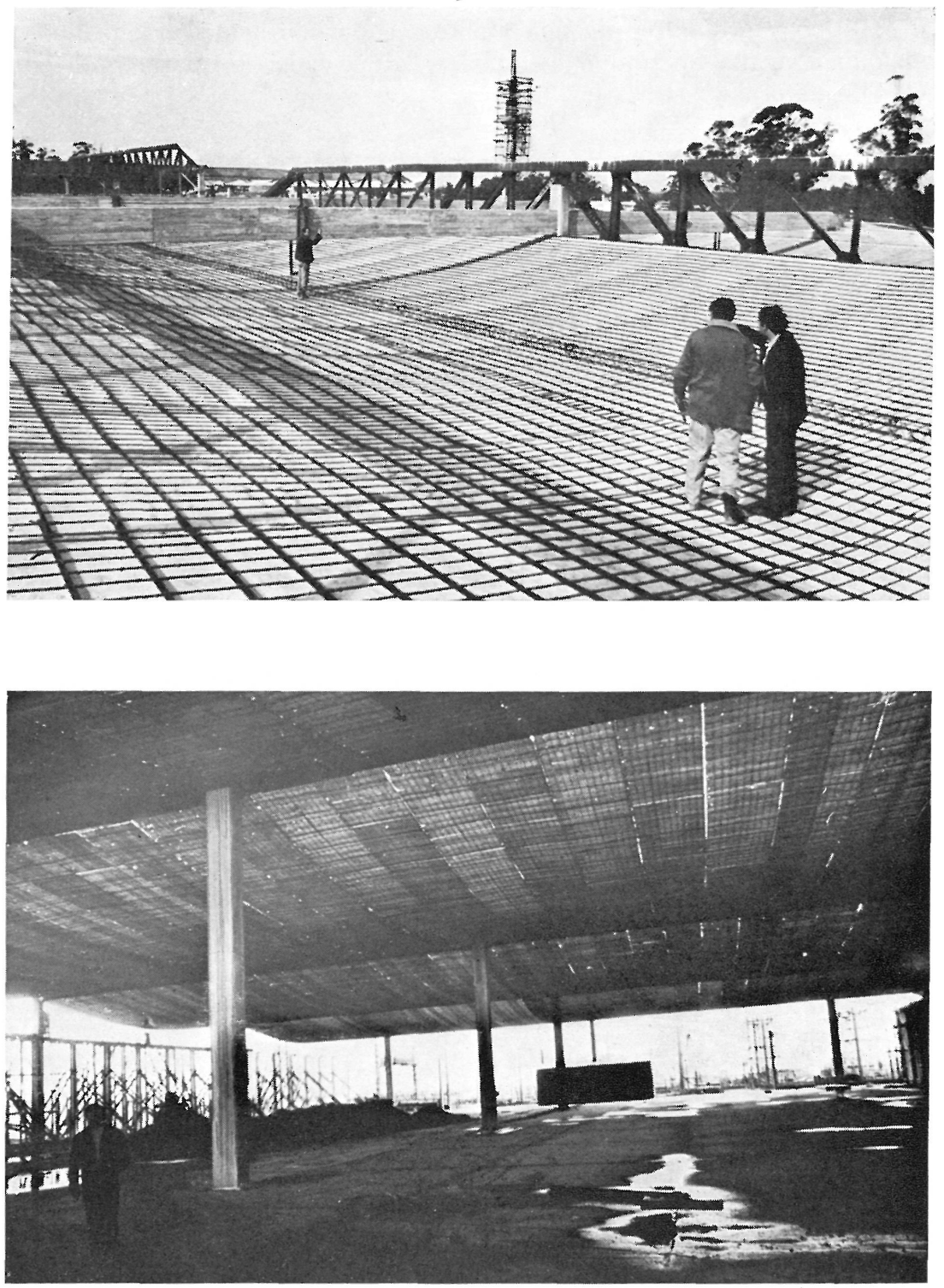

La segunda solución, de láminas colgantes de hormigón armado, se constituye con una estructura resistente formada por una malla metálica soldada. Dicha malla se prefabrica por sectores, utilizando redondos transversales y perfiles longitudinales, y fijándose al marco perimetral resistente, del que cuelga. Adopta una forma prismática a simple curvatura, con directriz de la catenaria transversal y generatrices rectas longitudinales que tienen una inclinación uniforme del orden del $1 \%$.

La malla se completa con paneles continuos y planchas de metal desplegado, fijados a ella con ataduras especiales. Ambos sirven de encofrado perdido para el hormigonado in situ de una capa de $3 \mathrm{~cm}$ de espesor, y actúan, además, como un efectivo aislante térmico y como base para la realización del cielorraso interior. El poliestireno expandido utilizado en estos paneles es uno de los mejores aislantes entre los que se emplean en la construcción y en la industria del frío, pudiéndose conseguir con él la eficacia requerida. 
El cielorraso interior, terminado con un salpicado a la cal, cumple varios propósitos, además del estético y protector: da una efectiva superficie anticondensante, de textura rugosa, que consigue una alta absorción acústica, y proporciona un paramento continuo que refleja la iluminación natural y artificial.

Exteriormente se realiza un alisado sobre la lámina de hormigón, para poder recibir en perfectas condiciones el techado hidráulico. La estructura laminar pétrea no trabaja por falta de momentos flectores, ya que las cargas de gravedad son absorbidas íntegramente por la malla metálica. Al aparecer sobrecargas no uniformes comienza a patentizarse la gran rigidez de su forma y de su estructura fuertemente armada, de aproximadamente $200 \mathrm{~kg}$ de hierro por metro cúbico de hormigón. Esto le permite absorber hasta cargas de una tonelada en mitad de una luz de $25 \mathrm{~m}$, produciéndose tan sólo una flecha de menos de $1 \mathrm{~cm}$. Gracias a ello pueden situarse libremente cargas puntuales, debidas a instalaciones auxiliares, que serán soportadas perfectamente.

El techado hidráulico deja de ser el punto crítico, como ocurre en la mayoría de las cubiertas de hormigón, porque aquí las láminas de base forman entre sí inmensos canales de desagüe, con pendientes en todos los sentidos, que evitan la acumulación del agua en cualquier punto. Se llega así a poder desaguar $11.000 \mathrm{~m}^{2}$ con sólo dos bajantes, lo que implica una gran economía en colectores e instalación general.

Todo el conjunto es muy estable por trabajar completamente a tracción y tener un peso propio que proporciona una total seguridad ante las succiones por viento y sobrecargas accidentales. A su vez, la simplicidad estructural permite desarrollar un cerramiento en mampostería de $0,15 \mathrm{~m}$ de espesor, sumamente efectivo y económico.

\section{résumé}

Couvertures suspendues en béton. République Argentine

Jorge W. Magaldi, ingénieur civil

Ces couvertures suspendues ont été le pour couvrir de grands espaces d'une manière appropriée et économique.

La structure porteuse de ces voiles es constituée par des cadres en béton armé. appuyant sur des fondations du même matériau. Ces cadres peuvent être préfabriqués, mai ils s'adapteront à tout moment pour chaque cas particulier.

Deux solutions de voiles suspendus peuvent être appliquées à la structure ainsi construite: l'une, métallique, et l'autre, en béton tes formes et matériaux complémentaires avec lesquels elles présentent des avantages d'étanchéité, entretien minimum, facilité pour la pose de l'isolement thermique et faux plafond, ainsi que la propriété de maintenir une hauteur libre intérieure presque uniforme couvrant de grands espatecturale de lignes très avancées.

\section{summary}

Concrete hanging trusses. The Argentine Republic

Jorge W. Magaldi, civil engineer

These hanging trusses are the result of a considerable number of studies on how to as economical manner.

The supporting structure is made up of reinforced concrete portals which rest upon portals can be prefabricated though in porticular case they have to to the special project in question.

The thus built structure is finished by means of two hanging sheets: one metal sheet and one of reinforced concrete. Both complementary materials whereby advantages are being obtained with regard to watertightnes, a minimum of maintenance easy placing of the thermal insulation and suspended ceiling, in addition to th possibility of maintaining an almost uniform face, and to throughout a great surdesign of very bold lines.

\section{zusammenfassung}

Hängedächer aus Stahlbeton. Die Argentinische Republik

Jorge W. Magaldi,

Diese Hängedacher sind das Ergebnis zahlreicher Studien, wie man grosse Flächen in ainer angemessenen un gleichzeitig

Die Tragkonstruktion dieser Folien besteht aus Stahlbetonportalen, die sich auf Gründungen desselben Materials stützen. Diese müssen sie in vorgefertigt sein; doch Projekt in Frage angepasst worden.

Die auf diese Art erbaute Struktur kann mittelst zwei hängender Folien abgeschlossen werden: einer Metallfolie und einer schiedene Formen und zusätzliche Materialien, wobei etliche Vorteile gewonnen werden, wie z.B. Wasserdichtigkeit, möglichst wenige Unterhaltung, leichte Verlegung der Wärmedämmung und der Hängedecke, ausser der Möglichkeit, bei grossen Flächen eine fast einheitliche innere lichte nischen Entwurf von sehr modernen Linien zu erlauben. 